\title{
CARCINOMA ERYSIPELOIDES OF THE BREAST IN A PATIENT WITH ADVANCED INVASIVE LOBULAR BREAST CANCER
}

\author{
Todor Yordanov ${ }^{1}$, Ivanka Temelkova ${ }^{1}$, Bogdana Ivanova $^{1}$, Elisaveta Popchristova ${ }^{2}$, \\ Neli Koleva ${ }^{2}$, Jenya Dimitrova ${ }^{3}$, Tsveta Kalinova ${ }^{3}$, Sonya Márina ${ }^{1}$ \\ ${ }^{1}$ Department of Skin and Venereal Diseases, \\ Medical Institute of the Ministry of Internal Affairs \\ ${ }^{2}$ Department of Clinical Pathology, Medical Institute of the Ministry of Internal Affairs \\ ${ }^{3}$ Department of Infectious Diseases, Parasitology and Dermatovenereology, \\ Faculty of Medicine, Medical University of Varna
}

\begin{abstract}
Carcinoma erysipeloides (CE) is an uncommon cutaneous metastasis of advanced breast carcinoma, showing distinctive features and presenting as a well-demarcated erythematous macule or plaque varying in size. It also may show a raised border and oedema. The differential diagnoses include erysipelas or cellulitis of the breast, or rarely-radiodermatitis. Most of the time $\mathrm{CE}$ is associated with advanced breast cancer and rarely with neoplastic tumours of the colon, pancreas, oesophagus, uterus, etc. We report a 58-year-old woman who presented at the Medical Institute of the Ministry of Internal Affairs with a 1-month history of not well-demarcated, erythematous, oedematous patches and plaques on the left and right breast, abdomen and both shins, associated with advanced invasive lobular carcinoma of the breast.
\end{abstract}

Keywords: carcinoma erysipeloides, metastatis, breast carcinoma

\section{INTRODUCTION}

Carcinoma erysipeloides (CE) is an uncommon cutaneous metastasis of advanced breast carcinoma. Most of the time it is associated with advanced breast cancer and rarely with neoplastic tumours of the colon, pancreas, oesophagus, uterus, etc (1).

Carcinoma erysipeloides usually presents with well-circumscribed, red to red-violasceous, oede-

Address for correspondence:

Tsveta Kalinova

Faculty of Medicine

Medical University of Varna

55 Marin Drinov St

9002 Varna

e-mail: immunefragment@gmail.com

Received: February 23, 2021

Accepted: March 15, 2021 matous skin resembling cellulitis. Usually the skin is tender, but also can be asymptomatic. Carcinoma erysipeloides is most commonly found on the presternal area of the body $(2,3)$. It may appear suddenly in normal skin and also within a scar from a previous surgery. In case of advanced breast cancer, it can be found on the other breast. Due to blocking of the draining lymphatics and the release of cytokines in the process, the patch or plaque can show a raised and oedematous border. Very recent review of the literature shows that cutaneous metastases of all kinds of tumours range between $0.7-10.0 \%(3,4)$. Cutaneous metastases are frequently found in women with breast cancer, rather than any other neoplasms. Rarely, CE can be the first sign of advancing breast cancer $(3,5)$.

Breast cancer is the second most common cancer among women, after basal cell carcinoma $(6,7)$. 
As mentioned above, $\mathrm{CE}$ is an uncommon type of cutaneous metastasis $(4,6)$. Inflammatory breast carcinoma was first described by C. Rasch in 1931 as carcinoma erysipeloides due to its similarity with erysipelas $(4,8)$. It constitutes a total of $1 \%$ of breast cancer metastases $(2,9)$. The differential diagnosis consists of erysipelas, cellulitis, candidiasis, radiodermatitis-like changes, etc. $(2,3,4)$. Clinically, carcinoma erysipeloides manifests with a well-demarcated, erythematous, tender area with raised, oedematous borders. In general, classic clinical features, lack of response to topical and systemic therapy should alert the physician to the possibility of cutaneous metastases (15). Tumour cells are within the dermal lymphatic vessels, with malignant thrombi-induced lymphatic obstructions causing the typical induration. The pathology of inflammatory carcinoma is specific, with the deposition of tightly packed neoplastic cells within the superficial and deep lymphatics without the pathologic evidence of acute inflammation. Dermal lymphatic invasion is considered to be the main hallmark of CE (11). Gross cystic disease fluid protein-15 (GCDFP-15) and estrogen receptor protein (ERP) are very specific and valuable markers for cutaneous metastatic invasive breast carcino$\mathrm{ma}$, which can be used in combination for better and more accurate diagnosis (12). As mentioned above, $\mathrm{CE}$ is an uncommon type of cutaneous metastatis. It is very rare compared to the more common papules, nodules, teleangiectatic, alopetic and zosteriform lesions (14).

\section{CASE REPORT}

A 58-year-old woman was presented to the Department of Internal Medicine, Pulmonology, Phtysiatry and Allergology with advancing bronchiectatic disease, decompensated chronic respiratory disease, pulmonary fibrosis, bilateral pleural effusions and oedema of the left hip, followed by the left shin, right axillary and left submandibular lymphadenomegaly. The laboratory evaluation showed: leucocytes-7.5 x $10^{9} / \mathrm{L}$; erythrocytes- $4.79 \times 10^{12} / \mathrm{L}$; haemoglobin- $136.0 \mathrm{~g} / \mathrm{L}$; haematocrit- $0.434 \mathrm{~L} / \mathrm{L}$; granulocytes-68.0\%; glucose-6.5 $\mathrm{mmol} / \mathrm{L}$; creatinine $-100.0 \mathrm{mmol} / \mathrm{L}$; urea $-5.6 \mathrm{mmol} / \mathrm{L}$; AST -88.0 IU/L; ALT-43.0 IU/L; LDH-264.0 IU/L; creatine phosphokinase-114.0 U/L; CK-MB fraction-29.0 IU/L; total protein-70.0 g/L; albumin-31.0 g/L;
GGT-135.0 IU/L; alkaline phosphatase-204.0 IU/L; amylase-94.0 IU/L.

A magnetic resonance tomography (MRT) of the head, neck, chest and abdomen regions was performed. It showed enlarged left submandibular, submental and axillary lymphadenomegaly, bilateral pleural effusion, pericardial effusion, and ascites. Finding in the uterus was indicative of carcinoma. There were generalized osteosclerotic findings.

A mammologic examination was perfomed. A formation in the upper left region of the right breast, $1 \mathrm{~cm}$ in diameter, suspected for breast carcinoma was found. A punch biopsy of the breast was initiated and histopathologic evaluation confirmed the diagnosis of invasive lobular breast carcinoma with perivasal infiltration (ER (3+), PR (3+), HER2(-)).

Two pleural punctures were performed in order to evacuate $1400 \mathrm{~mL}$ of pleural punctate that showed groups of large tumour cells.

An abdominal puncture was performed and $1500 \mathrm{~mL}$ of ascitic fluid was evacuated and no tumour cells were found.

The patient was referred to an oncology committee, so the newly diagnosed breast cancer can be evaluated and exact therapy can be initiated.

After TNM staging, a polychemotherapy, target therapy with Letrozole Nucleus $2.5 \mathrm{mg}$, and substitutional therapy were initiated.

Due to deterioration of the general condition of the patient, she was admitted to the hospital two times (at the Department of Internal Medicine, Pulmonology, Phtysiatry and Allergology). During her

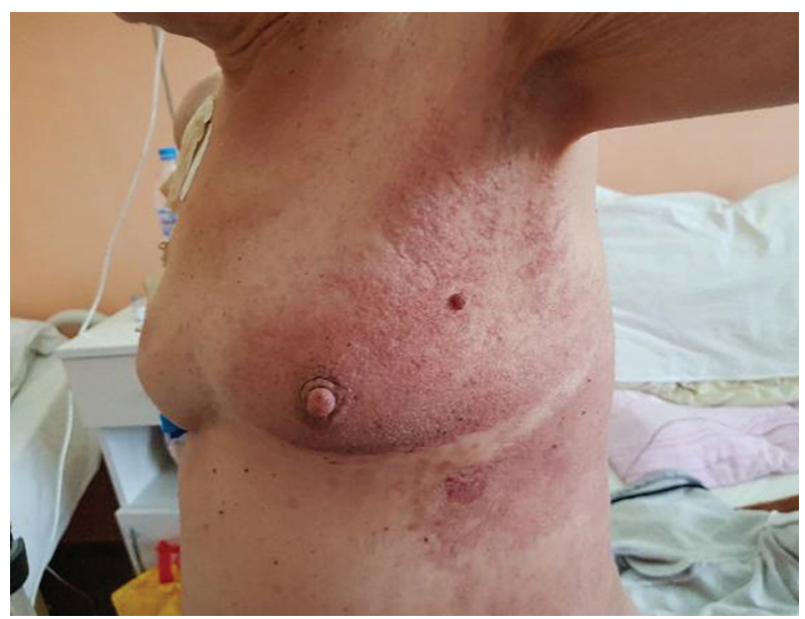

Fig. 1. Clinical presentation of the chest region 
Todor Yordanov, Ivanka Temelkova, Bogdana Ivanova et al.

second hospitalization two more pleural punctures were performed in order to evacuate $1100 \mathrm{~mL}$ of transudate.

During her last hospitalization in September 18 2020, a referral from a dermatologist was made. The patient complained of macules that appeared in the previous 2 weeks. The dermatological evaluation showed not-well-demarcated, erythematous, oedematous, tender patches with infiltration, very suspicious for carcinoma erysipeloides (Fig. 1, 2).

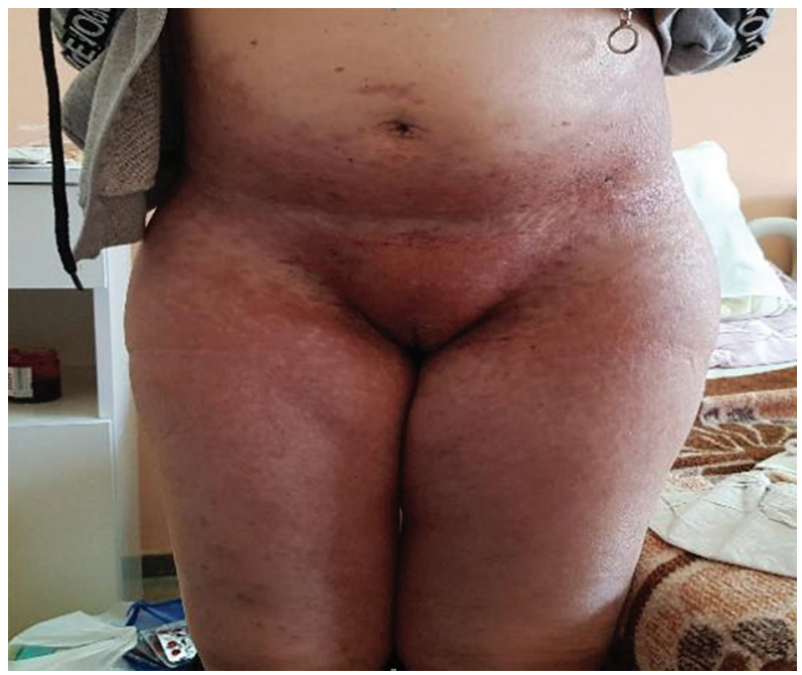

Fig. 2. Clinical presentation of the lower abdomen and hip region

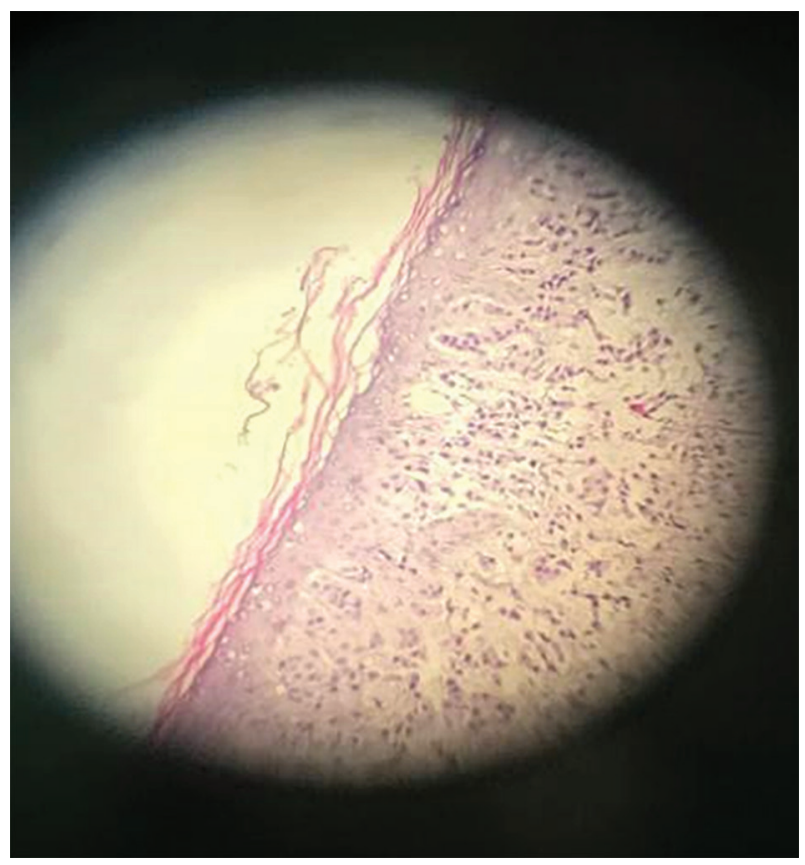

Fig. 3. Histological findings (x 100)
A 4-mm punch biopsy was performed. The histopathology of the skin showed massive infiltration of carcinomatous lymphangitis, probably from a breast carcinoma.

The histological evaluation of the biopsy specimen showed dysplastic cells predominantly growing within the superficial and deep dermal lymphatic vessels, with morphological features resembling inflammatory invasive breast carcinoma (Fig. 3, 4).

The laboratory tests showed: leucocytes-14.66 x $10^{9} / \mathrm{L}$, erythrocytes-3.17 $\times 10^{12} / \mathrm{L}$, haemoglobin-112.0 g/L, haematocrit-0.313 L/L, granulocytes-68.3\%, glucose-4.92 $\mathrm{mmol} / \mathrm{L}$, creatinin-92.1 mmol/L, urea-8.5 mmol/L, AST-167.0 IU/L, ALT-265 IU/L, LDH-264.0 IU/L, creatine phosphokinase-114.0 U/L, CK-MB fraction-29.0 $\mathrm{IU} / \mathrm{L}$, total protein-54.0 g/L; albumin-20.0 g/L, GGT-648.0 IU/L, alkaline phosphatase-667.0 IU/L, amylase-242.0 IU/L.

A MRT of the head, neck, chest and abdomen revealed three not-well-defined formations in the temporo-occipital region and medulla oblongata, suspected for metastases. Bilateral pleural effusion, right axillary lymphadenomegaly, tumour formation in left upper quadrant of the right breast, ascites and multifocal secondary osteoslerotic lesions were established.

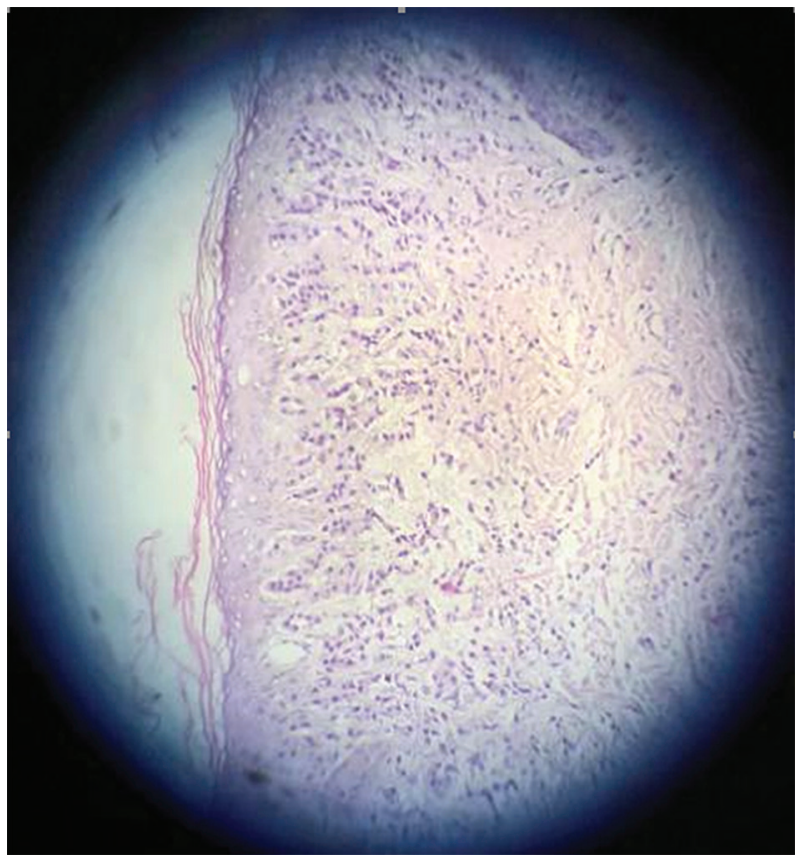

Fig. 4. Histological findings (x 200) 
In seven days, the patient's general condition deteriorated with psychomotoric agitation and multiorgan insuffiency leading to her death.

\section{DISCUSSION}

We presented a clinical case of a 58 -year-old woman with 1-month history for a rapidly evolving erythematous, oedematous and tender lesion on her left breast, abdomen and hips that appeared to be carcinoma erysipeloides. Our clinical case proves the need to maintain great suspicion for dermatitis- or erysipelas-like lesions over the breast, which are resistant to classical treatment. Awareness of the distinctive inflammatory manifestations of CE is essential for the physicians to establish an early diagnosis and accurate treatment in order to increase the overall survival rate.

\section{CONLCUSION}

Breast cancer is the second most common cancer among women. Rarely, this type of cancer can metastasize with atypical erythemous-oedematous plaques similar to infectious diseases of the skin (erysipelas or cellulitis). The right diagnosis is of a great importance for the treatment and survival of the patient. Our clinical case presents a female patient with erysipelas-like lesions on the skin, later diagnosed as advanced breast cancer. Through this clinical case we would like to underline the importance of rare differential diagnoses in clinical cases where the biological behaviour of the disease cannot be explained.

\section{REFERENCES}

1. Chisti MA, Alfadley AA, Banka N, Ezzat A. Cutaneous metastasis from breast carcinoma: A brief report of a rare variant and proposed morphological classification. Gulf J Oncol. 2013; 1(14):90-4.

2. Yun SJ, Park HY, Lee JS, Park MH, Lee JB, Won YH. Clinicopathological correlation of cutaneous metastatic breast carcinoma using lymphatic and vascular markers: lymphatics are mainly involved in cutaneous metastasis. Clin Exp Dermatol. 2012; 37(7):744-8. doi: 10.1111/j.1365-2230.2011.04306.x.
3. Nava G, Greer K, Patterson J, Lin KY. Metastatic cutaneous breast carcinoma: A case report and review of the literature. Can J Plast Surg. 2009; 17(1):25-7. doi: 10.1177/229255030901700105.

4. Rasch C. Carcinoma erysipelatoides. Br J Dermatol Syph. 1931; 43:351-4.

5. Eeskevari S, Gharaei, Nejad K. Carcinoma erysipeloides as the first manifestation of breast carcinoma: a case report. Iran J Dermatol. 2012; 15(3):102-4.

6. Gugle A, Malpathak V, Zawar V, Deshmukh M, Kote R. Carcinoma erysipeloides: an unusual presentation mimicking radiation dermatitis. Dermatol Online J. 2008;14(2):26.

7. Mordenti C, Peris K, Concetta Fangoli M, Cerroni L, Chimenti S. Cutaneous metastatic breast carcinoma. Acta Dermatoven APA. 2000; 9(4):143-8.

8. Anon. ABCDE "world cancer report". International agency for research on cancer. 2008.

9. Al Ameer A, Imran M, Kaliyadan G, Chopra R. Carcinoma erysipeloides as presenting feature of breast carcinoma: A case report and brief review of literature. Indian Dermatol Online J. 2015; 6(6):396-8. doi: 10.4103/2229-5178.169724.

10. Mortimer PS. Disorders of lymphatic vessels. In: Burns T, Breathnach S, Cox N, Griffiths C, editors. Rook's Text Book of Dermatology. 8th ed. London: Wiley-Blackwell; 2010. pp. 31-48.

11. Finkel LJ, Griffiths CE. Inflammatory breast carcinoma (carcinoma erysipeloides): An easily overlooked diagnosis. Br J Dermatol. 1993; 129(3):3246. doi: 10.1111/j.1365-2133.1993.tb11855.x.

12. Lookingbill DP, Spanger N, Helm KF. Cutaneous metastases in patients with monastic carcinoma: A retrospective study of 4020 patients. J Am Acad Dermatol. 1993; 29(2 Pt 1):228-36. doi: 10.1016/0190-9622(93)70173-q.

13. Canpolat F, Akpinar H, Eskioglu F, Genel N, Oktay M. A case of inflammatory breast carcinoma: Carcinoma erysipeloides. Indian J Dermatol Venereol Leprol. 2010; 76(2):215. doi: 10.4103/0378-6323.60543. 